\title{
TELOMERES AND TELOMERASE IN AGEING AND CANCER
}

\author{
John K. Cowell \\ Center for Molecular Genetics \\ Lerner Research Institute \\ Cleveland Clinic Foundation \\ 9500 Euclid Avenue \\ Ceveland $\mathrm{OH} 44195$
}

\begin{abstract}
Telomeres lie at the ends of human chromosomes and contain long tandem repeats of a simple nucleotide sequence. Because DNA replication cannot proceed to the very end of chromosomes, copies of these repeats are lost at each cell division. If the telomeres shorten below a critical length, the cells will eventually die as a result of genomic instability. Aging cells usually avoid death by entering senescence before the critical telomere length is reached. Malignantly transformed, immortal cells overcome senescence but they must still avoid the final, critical shortening of telomeres to survive. In the vast majority of cases, tumor cells achieve this by activating the telomerase enzyme, a ribonucleoprotein complex which repairs the end of chromosomes and prevents telomere shortening. Normal mortal cells do not normally express telomerase, although some stem cell populations which must regenerate thought the life span of the organism, retain enzyme activity. Cellular senescence can be overcome by inducing telomerase expression in mortal cells, firmly establishing the role of telomere length in the senescence signaling pathway. In tumor cells, the evidence of a role for telomerase in immortality is still largely correlative, with $\mathbf{8 0 - 9 0 \%}$ of tumors expressing telomerase activity. To establish whether telomerase activity is important in maintaining the malignant phenotype, attempts have been made to inactivate it in tumor cells, using a variety of approaches, where there is evidence that disrupting telomerase function can result in the induction of apoptosis. The background and implications of these observations is discussed.
\end{abstract}

\section{INTRODUCTION}

Telomeres are the specialized structures located at the ends of all eukaryotic chromosomes which ensure chromosome stability by protecting the ends of the chromosomes from degradation and end-to-end fusion $(1,2)$. When a chromosome arm lacks a telomere, it is more likely to be involved in end-to-end fusions and recombination which produces dicentric chromosomes. All eukaryotic telomeres consist of large numbers of simple tandem repeats. In human cells, the telomeres contain up to $15 \mathrm{~kb}$ of the tandem hexamer repeat,
TTAGGG (3). Because of the dynamics of the DNA replication fork mechanism, one strand of the double helix cannot be completely copied to the very end of the chromosome (4). As a result, a single strand gap is created at the end of each replication cycle and then a putative exonuclease is thought to remove the single strand overhang and this results in loss of approximately 100 bp of telomere repeats at each cell cycle. Thus, as normal cells continue to divide, their telomeres get shorter. Hastie and colleagues (5) demonstrated that this was clearly the case, since the telomeres from normal somatic cells from older individuals were shorter than in younger individuals. Thus, it was proposed that shortening of telomeres is an important event which contributes to the cellular aging process. The same phenomenon has also been shown in vitro, where human fibroblasts, for example, can normally undergo only a finite number of cell divisions, whereafter they enter a quiescent phase of so-called "replicative senescence". These cells also progressively lose telomere repeats as they continue to divide. The point at which these cells undergo senescence is referred to as the "M1 cell cycle checkpoint" or the Hayflick limit (6). Since this limit occurs consistently between experiments, after a fairly constant number of doublings, it was proposed that individual cells contain a "mitotic clock". This provides a cell division counting mechanism which, in turn, provides the signal to undergo senescence at the critical point. The correlation between telomere length and senescence implied that it may be the number of telomere repeats which are actually being counted (4). Senescent cells, however, are still viable cells and so they are capable of overcoming the M1 block, for example following infection by oncogenic viruses $(7,8)$, which then allows them to continue to divide for an additional, limited period whereafter they go into a more permanent senescence, termed "crisis" or the "M2 checkpoint". This crisis point is characterized by the type of karyotypic instability which has been observed for chromosomes lacking telomeres and results in significant cell death (9). Between the $\mathrm{M} 1$ and $\mathrm{M} 2$ checkpoints, the telomeres continue to shorten from approximately $5-7 \mathrm{~kb}$ to $2-4 \mathrm{~kb}$. During this period rare cells may emerge from the $M 2$ block which are now immortal. Telomere length in these cells now remains constant, indicating that the cells have acquired the ability to prevent further shortening of their telomeres. In the vast majority of cases this has been 
achieved by activating the telomerase enzyme (see below). The overall conclusion from these observations is that the lifespan of these cells is determined by the length of their telomeres. This same phenomenon has also been reported in yeast cells where mutations affecting telomerase function eventually result in telomere shortening and subsequent death if the inhibition is not overcome $(10,11)$.

\section{TELOMERASE AND CELLULAR AGING}

Telomerase is a ribonucleoprotein complex which allows the unreplicated end of chromosomes to be repaired into double strand DNA and so avoid further loss of telomeric repeats. The RNA component of telomerase carries a template for the telomeric repeat and part of the protein component of telomerase is a specialized reverse transcriptase which then copies this template. In this way, overhangs at the ends of chromosomes are repaired and telomere erosion is stopped (4). Although telomerase activity is re-established in cells escaping M2 crisis there are, however, also normal populations of cells which express telomerase and these cells tend to be derived from stem cell populations, for example, in the bone marrow (12), endothelial cells (13) and dermis of the skin (14), which must regenerate throughout the life of the organism. Germ line cells must also maintain their telomere length, since these same chromosomes must be passed to the next generation intact. It was not surprising, therefore, that these cells retain telomerase activity. With the exception of the germ cells, however, the telomeres of many of the normal cells which express telomerase, still shorten with age and so, apparently, do not express the enzyme at sufficiently high levels to completely maintain their telomeres. Furthermore, many cells induced to re-enter the cell cycle can also activate telomerase for the duration of the growth period (14-16). Thus, it is possible that there are threshold levels of telomerase activity below which not all telomeres can be maintained. This has important implications to the cell since, because telomere length heterogeneity is present amongst the individual chromosomes (17), it is possible that shortening of just a single telomere within the complement of chromosomes may be sufficient to trigger senescence.

The human telomerase holoenzyme contains an RNA molecule (hTR) which carries the template for telomere extension (18), and several essential proteins. One of these proteins, TP1/TLP, was cloned recently $(19,20)$, although the specific mechanism by which this protein contributes to the function of telomerase is not known. The reverse transcriptase component of telomerase, hTERT, has also been isolated $(21,22)$ which provided the opportunity to investigate the overall cellular control of telomerase expression. The cloning of hTERT also allowed the association between telomerase and life span to be analyzed further. Thus, when Bodnar et al (23) introduced the hTERT catalytic subunit into normal cells expressing hTR, it was shown that telomerase activity was recovered and their in vitro life span could be significantly extended beyond that seen in the untransfected counterparts. These cells also showed an extension of their telomere lengths.

The demonstration that inducing telomerase expression in normal cells can prevent shortening of their telomeres and extend their lifespan, strongly supports a central role for telomerase in the cellular aging process, although it is unlikely to be related to the overall aging of the organism. Even though manipulating telomerase may not help us live any longer, extending the lifespan of certain cells has obvious implications for the development of new therapeutic approaches for the treatment of certain diseases. Thus, for bone marrow transplantation, for example, being able to extend the lifespan of the cells grafted into the host may result in a more effective and permanent graft. Where genetically engineered cells can be used to complement a particular genetic deficiency, then possibly by increasing the longevity of these cells, the compensation for loss of function will also be extended. Clearly, characterizing how telomerase expression might overcome senescence is going to be very important in the future with many potential clinical applications. As well as being important in senescence, however, at the other end of the spectrum, telomerase also appears to be important in the establishment of immortality. These two events are clearly related since cancer is mostly a disease of old age. In fact, it has been suggested that it is the limitation on cell division in telomerase negative cells that results from telomere shortening, that may be an important anti-transformation mechanism used by the organism to prevent the continued expansion of premalignant clones of cells (24).

\section{TELOMERASE AND CANCER.}

The relationship between telomerase activity and malignant transformation is still largely correlative. Since tumor cells are rapidly dividing, they must overcome the disastrous consequences of telomere erosion. In fact, extensive studies $(25,26)$ have now shown that at least $85 \%$ of tumors achieve this by re-expressing telomerase. There also appears to be a correlation between tumor stage and telomerase activity. Thus, in low grade brain tumors, for example, telomerase activity is often undetectable, whereas in the very aggressive and malignant glioblastomas, telomerase expression is high (27). The very aggressive Stage 3 and 4 neuroblastomas show high levels of telomerase expression, but the variant form, called stage $4 \mathrm{~S}$, which often undergoes spontaneous regression, frequently does not show telomerase expression (28). Those stage $4 S$ tumors which do show telomerase expression often recur as more aggressive tumors. Thus, in the majority of tumors, telomerase appears to be activated to meet the demand of fast growing cells to maintain their telomeres. Activation of telomerase, however, is not the only mechanism by which telomere erosion is prevented, since certain 
cell lines, albeit relatively few, and some tumors, maintain large telomeres without telomerase activity (29). In the absence of any information about the underlying mechanism of this phenomenon, it has been referred to as the 'alternative' or ALT pathway. The commonly held belief is that some form of non-homologous recombination is responsible for maintaining telomere length in these cases.

A potentially important question about telomerase activity during malignant transformation, which might influence future strategies to target telomerase for anticancer therapy for example, concerns the mechanisms which controls reactivation of the enzyme. hTR does not seem to be the critical regulator, since hTR mRNA levels do not correlate with telomerase activity. In fact, many telomerase negative cells express hTR (30), sometimes at higher levels than telomerase positive cells. In addition, in cancer cells, even when telomerase levels increase dramatically, the levels of hTR show only minimal levels of increased expression (30). Thus, the control of the re-expression of telomerase activity is probably related to the protein components of the holoenzyme. The TP1/TLP gene is apparently not involved in regulation of enzyme activity since its presence or level of expression is not correlated with telomerase activity (19, 20). In contrast, Meyerson et al (21) demonstrated that, in fact, it is the activation of the hTERT component of telomerase which appears to regulate enzyme expression. Even despite hTR levels in some telomerasenegative normal tissues, hTERT mRNA was not detected, although it could be found in the majority of human cancer cells. In these cells hTERT mRNA levels correlated well with telomerase expression. During immortalization of normal cells by viral proteins, despite levels of hTR remaining constant, hTERT expression paralleled telomerase expression being absent in precrisis cells but present in post-crisis cells. Cells induced to undergo cellular differentiation switched off hTERT, which was correlated with loss of telomerase activity 24 hours later. The exact details of how hTERT is involved in regulation of telomerase expression have not yet been fully established, and it may prove to be more complex than at first thought, since there are clearly different splice variants of hTERT and more detailed studies will be required to understand their role in overall control of expression in cells from different origins.

\section{OF MICE.....}

The discovery of the ALT pathway demonstrated that telomerase is not essential for the maintenance of the malignant phenotype per se. But the question that arises is; why then do so many tumors re-express it? Clearly activation of telomerase in cancer cells occurs at a relatively late stage in the oncogenic process, when telomeres are already very short. This suggests that telomerase is only induced to prevent the final disastrous shortening events and, unless the cells can pre- vent complete telomere loss, they will die regardless of the other oncogenic events that are promoting the malignant phenotype.

To investigate this, and any other roles telomerase may have in the development of the organism, Blasco et al (31) created mice lacking the telomerase RNA (mTR). Cells from these mice lacked telomerase activity, but when mTR was reintroduced into cells from the null mice, telomerase activity was recovered. This suggests that the hTR can provide the signal to induce the protein components of the enzyme. However, some cells have endogenous expression of mTR, but do not induce the protein components for telomerase activity which suggests that the control of expression is more complex than a simple two-way interaction. When cells from mTR-deficient animals were grown in culture, both showed characteristics of senescence in early stages of in vitro growth, but both $\mathrm{mTR}$ - and $\mathrm{mTR}+$ cells could overcome the crisis phase and eventually immortalize, demonstrating that, in mice at least, telomerase is not necessary for immortalization. The telomeres in the mTR- cells shorten with successive cell division in vitro as expected. Furthermore, following transfection with oncogenic viral constructs, the mTR-deficient cells underwent malignant transformation at a rate equal to that of hTR + cells. These cells also produced tumors in nude mice. Thus, telomerase activity is not essential for these mouse cells to form tumors. As seen in human cells, however, even though the observation from the null mice demonstrated that this is not an essential event for transformation, most mouse tumor cells still activate telomerase. Again this raises the question why the tumor cells do this at all? Furthermore, mouse cells can also activate telomerase in the absence of critically shortened telomeres, suggesting telomerase activation is not in direct response to loss of telomeric repeats. One suggestion was that these cells may be activating telomerase in response to the increased proliferation rates in these cells. There are also suggestions that this is the case in human cells since, when keratinocytes (14) or bladder epithelial cells (15), for example, are forced into the cell cycle, they can be shown to transiently express telomerase, supporting the idea that telomerase activity may be related to the cell cycle.

The comparison of the regulation of telomerase activity between mice and man, however, should be viewed with some caution since, as Blasco et al (31) pointed out, there are considerable differences between normal mouse and normal human cells and specifically in their ability to transform. Thus, mouse cells are relatively easy to grown in vitro and transform spontaneously at a relatively high frequency. This is not the case for normal human cells which are often difficult to culture and rarely, if ever, transform spontaneously. These observations alone suggest that there may well be additional growth control checkpoints in human cells which are not present in mouse cells which, in turn, impose different controls on the regulation of telomerase activity (32). 
The role for telomerase in normal mouse cells which maintain a proliferative capacity throughout the lifespan of the organism was investigated by Lee et al (33) who demonstrated the need for telomerase in maintaining the normal cellular homeostasis at the biological and genomic levels. In these studies the reproductive system was studied in detail, since these cells depend on ordered cell expansion and development. After 6 generations (G6) of mice the number of litter mates declined drastically. The size of the testes in these animals was reduced by $80 \%$ compared with age-matched wild type animals, whereas organs such as the brain, for example, were unaffected. Approximately 60 cell doublings occur between the zygote and development of mature sperm and, in the G6 mice, there was a striking absence of spermatogenesis. The lack of sperm cells was due both to a decrease in proliferation as well as an increase in apoptosis. The consequences for the hematopoietic system were not so pronounced, although it appeared that long term renewal of stem cells is compromised by telomere loss, as was the mitogen-induced proliferation of primary splenocytes. The observations that maintenance of relatively normal blood cell counts, immune competence and gastrointestinal function, for example, is important and suggests that organ functions may not be compromised by using telomerase inhibitors in therapeutic applications.

\section{TELOMERASE AS A TARGET FOR CANCER THERAPY}

The strong correlative observation between re-expression of telomerase and the passage through the M2 crisis phase into full immortalization raised the question whether telomerase was absolutely required to maintain the malignant phenotype, at least in the vast majority of tumor cells which express it. To investigate this, Feng et al (18) transfected an antisense vector against the telomerase RNA into HeLa cells and demonstrated that cell proliferation in some clones ceased after approximately 23 doublings and their telomeres shortened. This suggests that further erosion of telomeres was involved in cell death. Other clones, however, appeared to be refractory to the treatment $(18,32)$. When the same experiment was performed in cells from gliomas (34), they went through a crisis phase but several clones emerged which were telomerase negative but still able to proliferate. The interpretation of these results has been difficult and the consequences of tumor suppression not yet fully investigated, although it is clear that inactivating telomerase can be a lethal event for some tumor cells.

In a more direct test, we have functionally and overwhelmingly inactivated the telomerase enzyme by selectively targeting and degrading $\mathrm{hTR}$, using a modified antisense oligonucleotide approach (35). The oligonucleotide used is a chimera between a modified phosphodiester antisense molecule and a 2'-5' oligoadenylate (2-5A) attached by a butanol linker. The
2-5A moiety is part of an RNA degradation pathway induced by the interferons $(36,37)$. In this system, $2-5 A$ synthetases generate the 2-5A molecule, which in turn activates a normally inactive endogenous RNAse $L$, resulting in RNA degradation $(38,39)$. Thus, when attached to an antisense oligo, which provides the specificity for the target RNA, the 2-5A component recruits RNAse $L$ to selectively degrade it (40-42). This system has been used in a variety of applications (40, $41,43,44)$, where it has been demonstrated to selectively degrade the target molecule. By selecting an open structure within the hTR target RNA, we have shown that hTR can also be targeted for degradation (27). Our initial studies have showed that when malignant brain tumor cells were treated in vitro with this antisense molecule, cell viability in the population was drastically reduced over a 7-10 day period (27). When cells are treated with control oligonucleotides, which either contain mismatches in the targeting sequence, or have the 2-5A moiety in the oligo inactivated, this effect is not seen. This series of experiments demonstrated that destroying hTR and inactivating telomerase causes relatively rapid cell death. Since these early experiments we have extended these observations and have shown that virtually all cell lines tested, which were derived from a number of different histological sites including breast, skin, kidney, prostate, liver and ovary, are affected in the same way. When normal human fibroblast and endothelial cells are treated with the same series of antisense molecules, there is no affect on cell viability. The tumoricidal effect was also seen in in vivo models in mice. Thus, when brain tumor cells were grown subcutaneously in nude mice and then treated by directly injecting the 2-5A antisense molecules into the tumor, tumor growth was suppressed and, in many cases, the size of the tumors was reduced compared with tumors treated with control oligos (27). We have since also shown that this same effect can be obtained with different tumor cell types. Thus, it appears that inactivating telomerase in these cells results in their death. The main effect on cell viability is seen over the first 2-4 days of treatment. This effect is apparently too immediate to be accounted for by an erosion of telomeres, since these cells clearly do not go through sufficient numbers of cell divisions before they die. However, as shown by Martens et al (17), not all telomeres are the same length and so it is still possible that shortening of just a single telomere could be the signal for cell death, although it appears more likely that inactivation of telomerase in some way triggers an intracellular surveillance pathway which commits the cells to die. Analysis of the tumor cells treated with 2-5A anti-hTR demonstrated that they undergo apoptosis both in vitro and in vivo (27), which accounts for the rapid cell death seen. Telomerase expression is clearly linked to the cell cycle, which in turn is linked to senescence and immortality and presumably the cellular homeostasis in these states is under tight control within human cells. It is possible, therefore, that telomerase is an important part of the surveillance checkpoint mecha- 
nisms used to monitor intracellular changes which may be detrimental to cell growth. Either through the physical act of degrading the RNA component of telomerase, or as a result of the sudden loss of telomerase function due to the degradation of hTR, an apoptosis response is induced. This response is not seen in normal cells treated with the 2-5A oligos and is not seen in tumor cells treated with control oligos, suggesting that it is a specific responses to the hTR damage. Since the majority of tumor cell lines we have been using are p53 deficient, the apoptosis seen does not appear to be dependent on an intact p53 checkpoint mechanism. The sudden loss of hTR, which occurs within 6 hours of oligo treatment (27), may also represent a different signal to that perceived by those cells where loss of telomerase function is more gradual, as seen in transfection experiments with the antisense vectors $(18,34)$. For now, the in vivo impact of targeting hTR with $2-5 \mathrm{~A}$ antisense is striking, with some tumors being reduced in size by $50 \% \mathrm{com}$ pared with tumors treated with control oligos. Although apoptosis appears to be responsible for this response in vivo as well, the molecular mechanisms of this response need to be explored more fully to gain a deeper insight into the role of telomerase in cellular homeostasis. It is possible that, by understanding the pathways involved in this phenomenon, even better anti-tumor therapies will be devised in the future.

\section{References}

1. Blackburn, EH: The molecular structure of centromeres and telomeres. Ann. Rev. Biochem., 53: 163-194, 1984.

2. Zakian, VA: Telomeres: beginning to understand the end. Science, 270: 1601-1607, 1995.

3. Moyzis, RK, Buckingham, JM, Cram, LS, Dani, M, Deaven, LL, Jones, MD, Meyne, J, Ratliff, RL, and Wu, JR: A highly conserved repetitive DNA sequence (TTAGGG)n, present at the telomeres of human chromosomes. Proc. Natl. Acad. Sci., 83: 6622-6626, 1988.

4. Greider, CW: Telomere length regulation. Ann. Rev. Biochem., 65: 337-365, 1996.

5. Hastie, ND, Dempster, M, Dunlop, MG, Thompson, AM, Green, DK, and Allshire, RC: Telomere reduction in human colorectal carcinoma and with ageing. Nature, 346: 866-868, 1990.

6. Hayflick, L, and Moorhead, PS: The serial cultivation of human diploid cell strains. Exp. Cell. Res., 25: 585-621, 1961.

7. Shay, JW, Pereira-Smith, OM, and Wright WE: A role for both $R B$ and $p 53$ in the regulation of human senescence. Exp. Cel Res., 196: 33-39, 1991.

8. Counter, CM, Avilion, AA, LeFeuvre, CE, Stewart, NG, Greider, CW, Harley, CB, and Bacchetti, S: Telomere shortening associated with chromosome instability is arrested in immortal cells which express telomerase activity. EMBO J., 11: 1921-1929, 1992.
9. Sack, GH: Human cell transformation by simian virus 40 - a review. In Vitro, 17: 1-19, 1981.

10. Counter, CM, Meyerson, M, Eaton, EN, and Weinberg, RA: The catalytic subunit of yeast telomerase. Proc. Natl. Acad. Sci. USA, 94: 92029207, 1997.

11. McEachern, MJ, and Blackburn EH: Runaway telomere elongation caused by telomerase RNA gene mutations. Nature, 376: 403-409, 1995.

12. Broccoli, D, Young, JW, and deLange, T: Telomerase activity in normal and malignant hematopoietic cells. Proc. Nati. Acad. Sci, USA, 92: 9082-9086, 1995.

13. Hsiao, R, Sharma, HW, Ramakrishnan, S, Keith, E, and Narayanan, R: Telomerase activity in normal human endothelial cells. Anticancer Res., 17: 827$832,1997$.

14. Harle-Bachor, C, and Boukamp, P: Telomerase activity in the regenerative basal layer of the epidermis in human skin and in immortal and carcinoma-derived skin keratinocytes. Proc. Natl. Acad. Sci. USA, 93: 6476-6481, 1996.

15. Belair, CD, Yeager, TR, Lopez, PM, and Reznikoff, CA: Telomerase activity: A biomarker of cell proliferation, not malignant transformation. Proc. Natl. Acad. Sci. USA, 94: 13677-13682, 1997.

16. Landberg, G, Nielsen, NH, Nilsson, P, Emdin, SO, Cajander, J, and Roos, G: Telomerase activity is associated with cell cycle deregulation in human breast cancer. Cancer Res., 57: 549-554, 1997.

17. Martens UM, Zijlmans JM, Poon SS, Dragowska W, Yui J, Chavez EA, Ward RK, and Lansdorp PM: Short telomeres on human chromosome 17p. Nature Genet., 18:76-80, 1998.

18. Feng J, Funk WD, Wang SS, Weinrich SL, Avilion AA, Chiu CP, Adams RR, Chang E, Allsopp RC, Yu J, Le S, West MD, Harley CB, Andrews WH, Greider $\mathrm{CW}$ and Villeponteau B: The RNA component of human telomerase. Science, 269: 1236-1241, 1995.

19. Harrington, L, McPhail, T, Mar V. Zhou, W, Oulton, R, Program, AE, Bass, MB, Arruda I, and Robinson, MO: A mammalian telomerase-associated protein. Science, 275: 973-977, 1997.

20. Nakayama, J-I, Saito, M, Nakamura, H, Matsuura, $A$, and Ishikawa, F: TLP: A gene encoding a protein component of mammalian telomerase is a novel member of WD repeats family. Cell, $88: 875-884$, 1997.

21. Meyerson, $\mathrm{M}$, Counter, $\mathrm{CM}$, Eaton, ENg, Ellisen, LW, Steiner, P, Dickinson Caddle, S, Ziaugra, L, Beijersbergen, RL, Davidoff, MJ, Qingyun, L, Bacchetti, S, Haber, DA, and Weinberg, RA: hEST2, the putative human telomerase catalytic subunit gene, is up-regulated in tumor cells and during immortalization. Cell, 90: 785-795, 1997. 
22. Kilian, A, Bowtell DDL, Abud, HE, Hime, GR, Venter, DJ, Keese, PK, Duncan, EL, Reddel, RR, and Jefferson, RA: Isolation of a candidate human telomerase catalytic subunit gene, which reveals complex splicing patterns in different cell types. Hum. Molec. Genet., 6: 2011-2019, 1997.

23. Bodnar, AG, Ouellette, M, Frolkis, M, Holt, SE, Chiu, CP, Morin, GB, Harley CB, Shay, JW, Lichtsteiner, S, and Wright, WE: Extension of lifespan by introduction of telomerase into normal human cells. Science, 279: 349-352, 1998.

24. Harley, CB, Kim, NW, Prowse, KR, Weinrich, SL, Hirsch, KS, West, MD, Bacchetti, S, Hirte, HW, Counter, CM, and Greider, CW: Telomerase, cell immortality, and cancer. Cold Spring Harb. Symp. Quant. Biol., 59: 307-315, 1994.

25. Kim, NW, Piatyszek, MA, Prowse, KR, Harley, CB, West, MD, Ho, PL, Coviello, GM, Wright, WE, Weinrich, SL, and Shay, JW: Specific association of human telomerase activity with immortal cells and cancer. Science, 266: 2011-2015, 1994.

26. Shay, JW, and Bacchetti, S: A survey of telomerase activity in human cancer. Eur. J. Cancer, 33: 787791, 1997.

27. Kondo S, Tanaka $Y$, Kondo $Y$, Hitomi M, Barnett $\mathrm{GH}$, Ishzaka $Y$, Liu J, Haqqi T, Nishiyama A, Villeponteau B, Cowell JK, Barna BP: (1998) Antisense telomerase treatment: Induction of two distinct pathways, Apoptosis and Differentiation. FASEB. J. 12, 801-811.

28. Hiyama, E, Hiyama, K, Yokohama, T, Mmitsuura, $Y$, Piatysek, MA and Shay, JW: Correlating telomerase activity levels with human neuroblastoma outcomes. Nature Medicine. 1: 249-255, 1995.

29. Bryan, TM, Englezou, A, Dalla-Pozza, L, Dunham, MA, Redell, RR: Evidence for an alternative mechanism for maintaining telomere length in human tumors and tumor-derived cell lines. Nature Medicine, 3: 1271-1274, 1997.

30. Avilion, AA, Piatyszek, MA, Gupta, J., Shay, JW, Bacchetti, S, and Greider, CW: Human telomerase RNA and telomerase activity in immortal cell lines and tumor tissues. Cancer Res., 56: 645-650, 1996.

31. Blasco, MA, Lee, H-W, Hande, MP, Samper, E, Lansdorp, PM, DePinho, RA, and Greider, CW: Telomere Shortening and Tumor Formation by Mouse Cells Lacking Telomerase RNA, Cell,91: 2534, 1997.

32. Harley, CB, and Sherwood SW: Telomerase, checkpoints and cancer. Cancer Surveys, 29: 263-284, 1997.

33. Lee, H-W, Blasco, MA, Gottlieb, GJ, Horner, JW, Greider, CW, and DePinho, RA: Essential role of mouse telomerase in highly proliferative organs. Nature, Vol. 392: 569-574, 1998.
34. Kondo S, Tanaka $Y$, Kondo $Y$, Hitomi M, Barnett $\mathrm{GH}$, Ishizaka $Y$, Liu J, Haqqi T, Nishiyama A, Villeponteau B, Cowell JK and Barna BP: Antisense telomerase treatment; induction of two distinct pathways, apoptosis and differentiation. FEBSJ.

35. Silverman $\mathrm{RH}$. 2-5A dependent RNase L: A regulated endoribonuclease in the interferon system. In, "Ribonuclease: structure and function", (eds. G. D'Alessio and J.F. Riordan), Academic Press, Inc., 1997.

36. Kerr IM and Brown RE: pppA2'p5'A2'p5'A: An inhibitor of protein synthesis synthesized with an enzyme fraction from interferon-treated cells. Proc. Natl. Acad. Sci. USA, 75: 256-260, 1978.

37. Johnston MI and Torrence PF. In; Interferon vol. 3 (ed. Friedman RM) pp. 189-298, Elsevier Science, New York, 1984.

38. Clemens MJ and Williams BRG: Inhibition of cellfree protein synthesis by pppA2'p5'A2'p5'A: A novel oligonucleotide synthesized by interferon-treated cell extracts. Cell, 13: 565-572, 1978.

39. Zhou A, Hassel BA and Silverman RH: Expression cloning of 2-5A-dependent RNAase; a uniquely regulated mediator of interferon action. Cell, 72 : 753-765, 1993.

40. Torrence, PF., Maitra, RK., Lesiak, K., Khamnei, S., Zhou, A., and Silverman, RH: Targeting RNA for degradation with a (2'-5') oligoadenylate-antisense chimera. Proc. Natl. Acad. Sci. USA, 90: 13001304, 1993.

41. Maran, A., Maitra, RK., Kumar, A., Dong, B., Xiao, W., Li, G., Williams, BRG., Torrence, PF., and Silverman, $\mathrm{RH}$ : Blockage of NF-kB signaling by selective ablation of an mRNA target by $2-5 \mathrm{~A}$ antisense chimeras. Science, 265: 789-792, 1994.

42. Hassel, BA., Zhou, A., Sotomayor, C., Maran, A., and Silverman, $\mathrm{RH}$ : A dominant negative mutant of 2-5A-dependent RNase suppresses antiproliferative and antiviral effects of interferon. EMBO J., 12: 3297-3304, 1993.

43. Maitra, RK., Li, G., Xiao, W., Dong, B., Torrence, PF., and Silverman, $\mathrm{RH}$ : Catalytic cleavage of an RNA target by 2-5A antisense and RNase L. J. Biol. Chem., 270: 15071-15075, 1995.

44. Cirino, NM., Li, G., Xiao, W., Torrence, PF., and Silverman, $\mathrm{RH}$ : Targeting RNA decay in respiratory syncytial virus infected cells with $2^{\prime}->5^{\prime}$ oligoadenylate-antisense. Proc. Natl. Acad. Sci. USA, 94:1937-1942, 1997. 